\title{
Developments in radiation techniques for thoracic malignancies
}

\author{
Tobias Finazzi $\mathbb{1}^{1}$, Famke L. Schneiders ${ }^{2}$ and Suresh Senan ${ }^{2}$ \\ Number 3 in the Series "Thoracic oncology" \\ Edited by Rudolf Huber and Peter Dorfmüller
}

${ }^{1}$ Clinic of Radiotherapy and Radiation Oncology, University Hospital Basel, Basel, Switzerland. ${ }^{2}$ Dept of Radiation Oncology, Amsterdam University Medical Centers, Location VUmc, Amsterdam, The Netherlands.

Corresponding author: Tobias Finazzi (tobias.finazzi@usb.ch)

Shareable abstract (@ERSpublications)

Technical advances have led to a changing perception of the role of radiation therapy in multidisciplinary care of lung cancer. This article provides an overview of recent developments in radiation therapy as a cornerstone of modern lung cancer treatment. https://bit.ly/34YTAtP

Cite this article as: Finazzi T, Schneiders FL, Senan S. Developments in radiation techniques for thoracic malignancies. Eur Respir Rev 2021; 30: 200224 [DOI: 10.1183/16000617.0224-2020].

Copyright @ERS 2021

This article is open access and distributed under the terms of the Creative Commons Attribution Non-Commercial Licence 4.0.

Received: 10 July 2020 Accepted: 27 Oct 2020

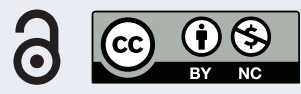

\section{Abstract}

Radiation therapy is a cornerstone of modern lung cancer treatment alongside surgery, chemotherapy, immunotherapy and targeted therapies. Advances in radiotherapy techniques have enhanced the accuracy of radiation delivery, which has contributed to the evolution of radiation therapy into a guidelinerecommended treatment in both early-stage and locally advanced nonsmall cell lung cancer. Furthermore, although radiotherapy has long been used for palliation of disease in advanced lung cancer, it is increasingly having a role as a locally ablative treatment in patients with oligometastatic disease.

This review provides an overview of recent developments in radiation techniques, particularly for nonradiation oncologists who are involved in the care of lung cancer patients. Technical advances are discussed, and findings of recent clinical trials are highlighted, all of which have led to a changing perception of the role of radiation therapy in multidisciplinary care.

\section{Introduction}

Lung cancer accounted for more deaths than breast, prostate and colorectal cancers combined in 2020 [1]. However, data from the Surveillance, Epidemiology and End Results (SEER) programme revealed that declines in mortality had accelerated for lung cancer in the period from 2008 to 2017, a finding which may be related to the rapidly evolving treatment landscape for lung cancer, especially for nonsmall cell lung cancer (NSCLC). In the past decade, molecular targeted therapies, immune checkpoint inhibitors and improved high-precision radiation delivery have joined the established modalities of chemotherapy, large-field radiotherapy and surgery in thoracic disease management. With the availability of new treatment options, the role of multidisciplinary tumour boards has become crucial in selecting and tailoring treatment strategies, and for managing toxicity and survivorship issues.

Updated guidelines have incorporated many of the incremental improvements in radiotherapy planning and delivery [2-4]. There are now more standardised definitions of target volumes, improvements in radiotherapy plan quality (including daily on-table treatment plan revisions) and reductions in organ at risk doses, all of which have increased clinician confidence to deliver ablative doses of radiation. Changes in thoracic radiotherapy guidelines [5-7] have partly been driven by results of studies showing improvements in population outcomes following the implementation of new radiotherapy techniques [8-12]. Recent prospective trials in early-stage lung cancer [13], locally advanced NSCLC [14, 15] and oligometastatic (lung) cancer [16-20] have also contributed to a changing perception of the role of radiation in multidisciplinary care. 
This review aims to provide an overview of recent developments in radiation techniques for non-radiation oncologists involved in the care of patients presenting with thoracic malignancies.

\section{Radiation therapy for early-stage lung cancer}

Nearly 25 years ago, the first clinical experience with so-called stereotactic radiation therapy for lung tumours was reported [21]. This technique, now termed stereotactic ablative radiotherapy (SABR) or stereotactic body radiation therapy, has since become the preferred treatment for early-stage NSCLC in patients who are medically inoperable or unwilling to undergo surgery [2, 3, 5]. Briefly, SABR is an approach used to deliver high radiation doses to tumours outside the brain, by using precise techniques to ensure that the tumour position is reproducible, while sparing critical surrounding normal tissues from toxicity. Curative treatments can then be delivered in fewer fractions than with use of conventionally fractionated radiotherapy, as on-board imaging can verify the tumour position before each session (figure 1).

The superior tumour control rates with lung SABR compared to conventionally fractionated radiotherapy have been demonstrated in large institutional series, as well as two randomised trials, one of which also showed improved overall survival with SABR [13, 22]. Lung SABR is typically delivered in between one and eight fractions during a period of up to 2 weeks in an outpatient setting. For selected peripherally located lung tumours, SABR can be delivered in a single session, based on the findings of two randomised studies in early-stage NSCLC showing outcomes comparable to SABR delivered in three or four fractions $[23,24]$. Local control rates with SABR can exceed 90\% for early-stage NSCLC and are generally considered comparable to those achieved with sublobar resection [25-27]. Regular computed tomography (CT) imaging is recommended after SABR in order to distinguish common patterns of focal fibrosis (figure 1) from so-called high-risk features that are suspicious for recurrence [28, 29]. Multidisciplinary assessment of radiological changes that evolve with time may aid in reassuring patients and also avoid unnecessary invasive procedures. Furthermore, multidisciplinary review is necessary for coordinating salvage treatment options in patients with a confirmed post-SABR recurrence [30].

Prospective data comparing SABR to lobectomy, the current standard of care in stage I NSCLC, are limited [31], but randomised trials comparing SABR to surgical resection are ongoing [32]. With the population of elderly patients with lung cancer increasing rapidly, it is relevant to note that SABR is well tolerated in unfit patients with multiple comorbidities, including severe COPD [2]. Elderly patients presenting with an early-stage NSCLC show an improved survival with SABR compared with observation alone [8]. A Dutch population study demonstrated increases in survival of elderly patients with stage I NSCLC following the introduction of SABR, which was also associated with a decrease in the numbers of patients who were untreated [9]. Following the introduction of SABR, significant changes in patterns of care for the elderly were observed in the Dutch population-based study, with $84 \%$ of patients aged $\geqslant 80$ years now undergoing SABR for stage I NSCLC [33]. However, patient groups who are at increased risk for SABR-related toxicity have also been identified, such as those with tumours abutting central
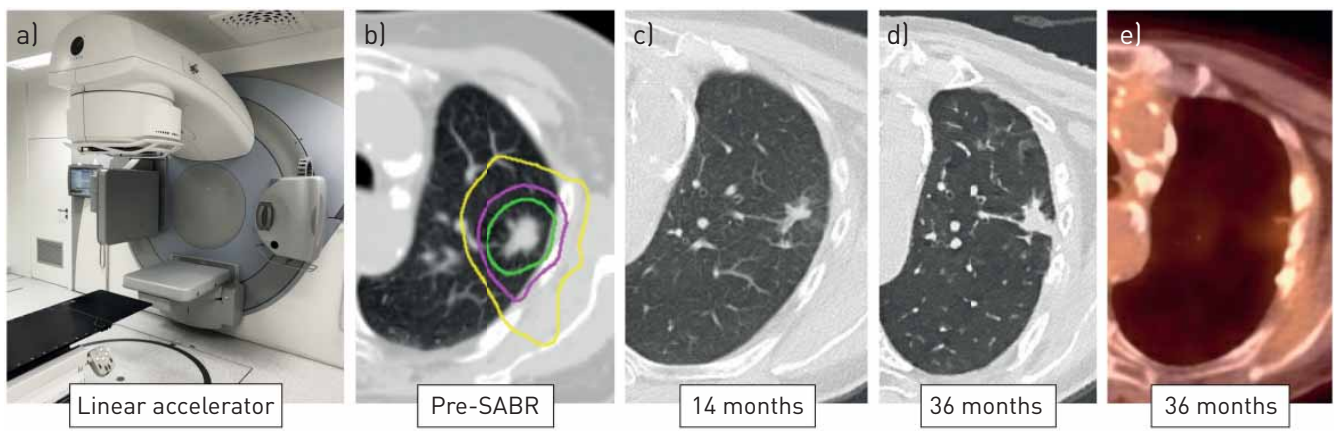

FIGURE 1 Serial diagnostic images of a peripheral stage I nonsmall cell lung cancer treated using a) stereotactic ablative radiotherapy (SABR) to a dose of $55 \mathrm{~Gy}$, delivered in five fractions, on a linear accelerator. An on-board cone-beam computed tomography (CT) scan is used for the daily on-couch verification of tumour position. b) The planned dose distribution is illustrated using so-called isodose lines, representing 50\% (yellow), $75 \%$ (purple) and 100\% (green) of the prescribed radiation dose. High-precision dose delivery to the tumour minimises irradiation of surrounding normal tissues. c) Follow-up images at 14 months reveal ground-glass changes and focal fibrosis in the treated region. d, e) At 36 months, residual scarring is present, with a positron emission tomography-CT showing no residual $18 \mathrm{~F}$-fluorodeoxyglucose uptake in the lesion. 
airways [34-37], with large tumours [38], or patients with coexisting interstitial lung disease (ILD) [39, 40]. For patients who are at a higher risk of surgical complications, SABR can offer a lower risk alternative, both for primary and recurrent NSCLC [41]. Patients undergoing surgery for a second NSCLC were found in a meta-analysis to have a pooled operative mortality of $7 \%$ for the second resection and a pooled 5 -year overall survival of only 46\% [42]. Life-long follow-up after any initial treatment for lung cancer is supported by population studies showing that the post-surgical cumulative risk of second primary lung cancer is $20 \%$ at 10 years post-surgery and $25 \%$ at 14 years post-surgery [43]. In patients undergoing SABR for early-stage NSCLC, distant (or out-of-field) recurrences remain the most common pattern of failure [44]. In order to reduce the risk of distant recurrences, combinations of immune checkpoint inhibitors and SABR in the early-stage or locally recurrent setting are currently being evaluated in phase II (e.g. I-SABR) and phase III (e.g. PACIFIC-4 and KEYNOTE-867) clinical trials [45-47]. The rationale supporting immune-SABR involves preclinical studies showing that the immunomodulatory effects of short-course radiotherapy can enhance both local and systemic antitumour immune response [48].

Further improvements in SABR delivery are desirable, for example by using techniques such as intensity-modulated radiation therapy (IMRT), an approach which allows for reductions in dose to critical organs such as the heart, lungs and oesophagus [49-51]. Volumetric modulated arc therapy (VMAT) is an approach which allows for the faster delivery of IMRT, which is now recommended in clinical practice guidelines for SABR [2]. SABR can also be delivered using proton beams, a technique where charged particles are used to deposit their energy more precisely in the tumour, thereby potentially reducing radiation doses to normal tissues. However, proton-based lung SABR is technically challenging as respiration and cardiac-induced tumour motion can degrade the quality of proton treatment plans even more than with conventional beams $[52,53]$. Proton-based lung SABR is currently undergoing evaluation in clinical trials [54-56].

Better tumour targeting in SABR can improve tumour control rates, and different approaches have been endorsed in clinical practice guidelines [2, 4]. A recommended strategy for lung tumours involves the use of a 4-dimensional (4D) CT scan for treatment planning, and all observed motion is used to generate a so-called internal target volume (ITV). However, in cases where tumours exhibit significant motion, the ITV approach can expose the healthy lung to a higher radiation dose [57]. Daily pre-treatment verification of the tumour position is mandatory [2], and this is commonly performed using integrated cone-beam CT technology on a conventional linear accelerator. However, as tumour motion during treatment delivery can exceed that observed on pre-treatment 4D-CT imaging [58, 59], motion monitoring during SABR delivery is desirable. Respiration-gated radiotherapy refers to radiation delivery that is restricted to a predefined tumour position and/or phase of the patient's respiratory cycle. Another approach in clinical use is tumour tracking, where the treatment beam continuously follows or is shaped to the tumour position [57]. Such so-called active motion management approaches can permit use of smaller treatment volumes [60-63]. However, motion management involving implantation of fiducial markers [64] or electromagnetic transponders [65] carries risks, especially in the elderly and frail patients [64, 66, 67]. Noninvasive monitoring of respiratory movements is possible using spirometric devices [68] or infrared or optical cameras to monitor surface breathing motion [68, 69], although these approaches can fail to accurately capture variations in tumour position [70].

Recent advances in radiotherapy technology aim to circumvent the need for implanted fiducials, or other surrogate markers, to capture tumour motion in real-time. Since 2014, direct tumour visualisation during radiation delivery has become possible with use of so-called magnetic resonance-guided radiotherapy devices. These hybrid machines incorporate both magnetic resonance imaging and radiotherapy technology into a single treatment system, which allows for continuous acquisition of magnetic resonance images during treatment [71, 72]. On-board magnetic resonance technology facilitates tumour gating without additional radiation exposure, and enables daily on-table plan adaptation, which improves treatment plans if doses to the tumour and/or critical organs are suboptimal due to changing anatomy. Magnetic resonance-guided lung SABR can be delivered with high precision [73], to significantly smaller target volumes than with a traditional motion-encompassing ITV approach (figure 2) [60]. In addition, the safety of SABR delivered to tumours in the proximity of critical organs at risk is improved using magnetic resonance guidance (figure 3) [74-77].

Developments such as magnetic resonance-guided radiation therapy, or proton therapy, serve to illustrate more complex techniques that are required to further improve treatment accuracy and reduce doses to healthy tissue. The latter becomes more important when performing so-called hypofractionation, which refers to the delivery of large radiation doses in a shorter overall treatment time. Hypofractionation, including single-fraction treatment, is being encouraged for reasons including better resource utilisation, 


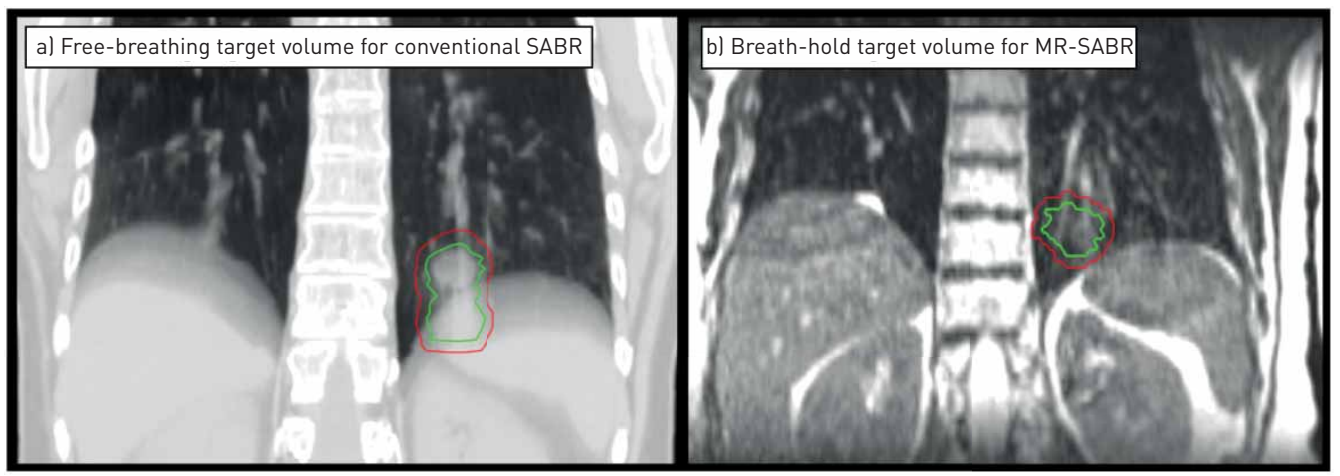

FIGURE 2 Planning images derived from a patient treated with magnetic resonance-guided stereotactic ablative radiotherapy (SABR) for a left lower lobe tumour. a) A motion-encompassing target volume (red contour) derived from a planning four-dimensional computed tomography scan for free-breathing conventional SABR. b) The actual target volume (red contour) when a pre-treatment breath-hold magnetic resonance (MR) scan was acquired. The smaller target volume for magnetic resonance-guided SABR avoids risk of high-dose delivery to the stomach, and accurate delivery to smaller target volumes is ensured by continuous target tracking and automated beam triggering.

patient comfort and easier scheduling of SABR treatments in patients with oligometastases in whom systemic therapies cannot be interrupted for long [78]. In the coronavirus disease 2019 (COVID-19) era, the European Society for Radiotherapy and Oncology (ESTRO) and the American Society for Radiation Oncology (ASTRO) have also recommended performing single-fraction lung SABR for stage I

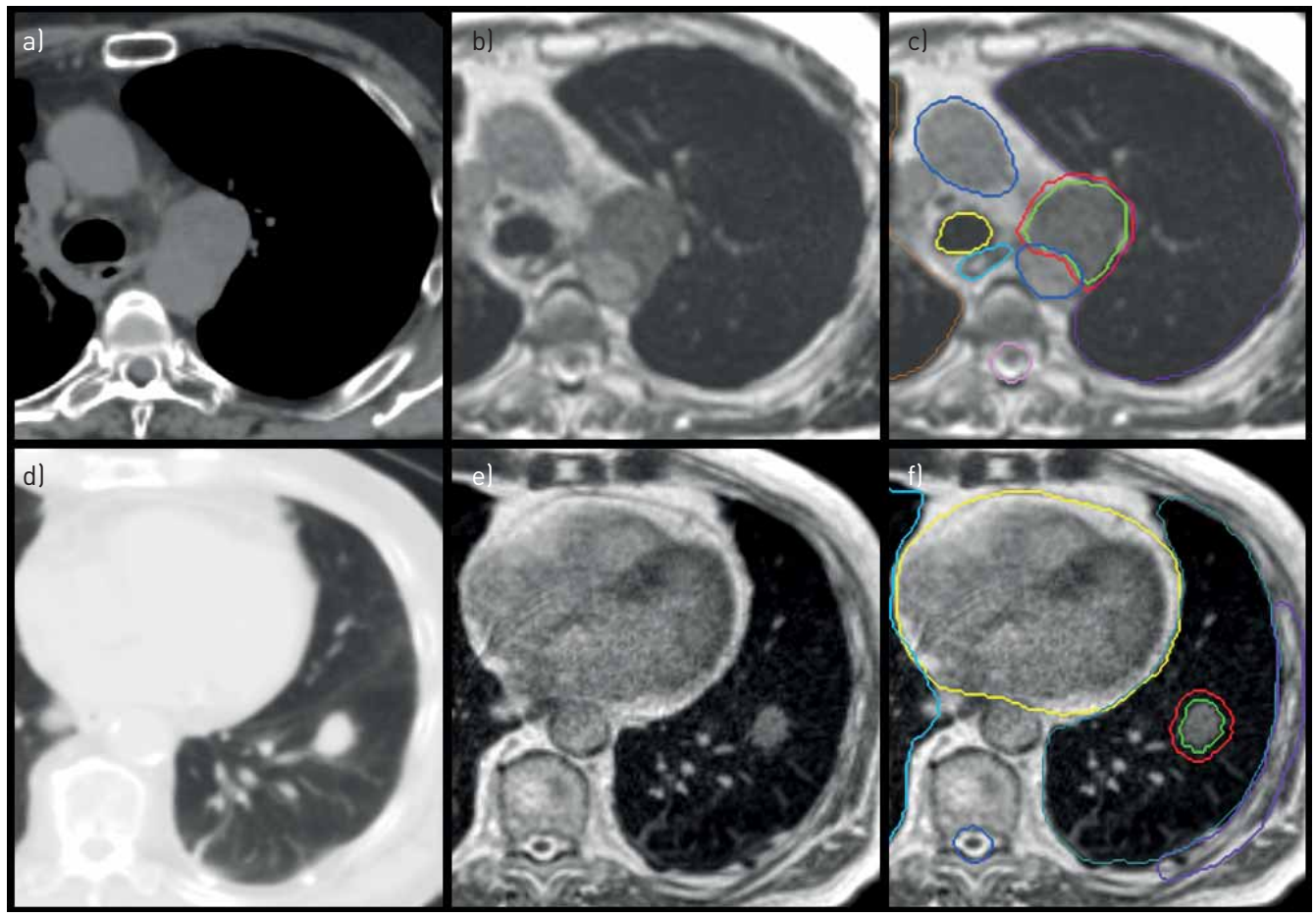

FIGURE 3 Axial panels of two patients treated using real-time magnetic resonance-guided stereotactic ablative radiotherapy, one for $a-c$ ) centrally located recurrence and one for $d-f$ ) peripheral early-stage nonsmall cell lung cancer. a, d) Computed tomography imaging represents the standard imaging modality for lung tumours. b, e) Linear accelerators with on-board magnetic resonance imaging allow for improved visualisation of soft tissues. c) The daily adaptation of radiotherapy treatment plans allowed for the minimisation of toxicity risks for a tumour recurrence in the mediastinum, which was treated in eight fractions to $60 \mathrm{~Gy}$. f) A peripheral lung tumour located at a non-critical location, which was treated using a single fraction of $34 \mathrm{~Gy}$. 
peripherally located NSCLC [7], and developments such as magnetic resonance guidance can increase clinician confidence in doing so [79]. However, such resource-intensive procedures are best suited for high-risk tumours, such as those in the hilar region, tumours arising after prior lung radiotherapy and when coexisting ILD is identified [80]. This technology is new, and technical challenges remain as magnetic resonance resolution is limited by respiratory and cardiac motion, and by the low signal-to-noise ratio of lung parenchyma [81].

\section{Locally advanced lung cancer}

Locally advanced NSCLC comprises a heterogeneous group of patients, including tumours with advanced local infiltration (T4), or ipsilateral (N2) and contralateral (N3) mediastinal lymph node metastases, among other criteria. These stage III tumours make up 35\% of all NSCLC cases at diagnosis [82]. The management of stage III NSCLC is complex and requires assessment by a multidisciplinary team. Many patients with stage III NSCLC are considered to be unresectable due to the extent of disease, even after induction therapy. However, in potentially resectable stage IIIA NSCLC, the standard of care should include consideration of surgical resection after multidisciplinary review [82, 83]. The overall prognosis of locally advanced NSCLC patients is poor even in resectable patients, for whom a 5-year overall survival of only $29.0 \%$ and $13.0 \%$ was observed for stage IIIA and IIIB disease, respectively [84].

Treatment paradigms for patients with inoperable stage III NSCLC have evolved in recent decades, from sequential to concurrent chemotherapy and radiotherapy (chemoradiotherapy; CRT), with the recent addition of consolidation immunotherapy as a standard of care in non-progressing patients [85]. Concurrent platinum-based CRT is the recommended treatment backbone for fit patients with unresectable stage IIIA and IIIB disease, as it improves 5-year overall survival compared to sequential CRT [86]. In the recent PACIFIC trial, 12 months of immune checkpoint inhibitor therapy with durvalumab, a monoclonal antibody targeting programmed death-ligand 1, improved overall survival compared to placebo in patients who did not progress following CRT. Updated follow-up at a median of 34.2 months revealed a median overall survival of 47.5 months in the durvalumab arm, as compared to 29.1 months in the placebo arm $[14,15,87]$.

Guidelines by the European Society for Medical Oncology for concurrent CRT recommend the delivery of 60-66 Gy in 30-33 daily fractions of 2 Gy, with the maximum overall treatment time not exceeding 7 weeks [5]. The dose of $60 \mathrm{~Gy}$ is considered a standard based on the Radiation Therapy Oncology Group (RTOG) 0617 study, in which a higher dose of 74 Gy was associated with worse overall survival [88]. Although modern radiotherapy techniques may allow for safer dose escalation to, for example, $66 \mathrm{~Gy}$, this is generally not recommended in patients with extensive nodal disease due to increased oesophageal toxicity. In both concurrent and sequential CRT, the choice of chemotherapy regimen is based on histology [82]. Concurrent CRT is associated with higher acute oesophageal toxicity rates (grade 3-4) compared to sequential CRT (18\% versus 4\%) [86]. Patients who are considered unsuitable for concurrent CRT, for example due to patient frailty or extent of disease, are candidates for sequential CRT, which is a valid alternative according to European Society for Medical Oncology guidelines [5, 85]. For sequential CRT, the radiotherapy scheme is preferably delivered in a shorter overall treatment time using hypofractionated regimens. Such shorter treatments, e.g. delivery of $55 \mathrm{~Gy}$ in 20 fractions of $2.75 \mathrm{~Gy}$ [89], appear desirable in the COVID-19 era, during which the treatment of patients with stage III NSCLC can be challenging, but should not be delayed [90]. However, the ESTRO/ASTRO consensus recommended that hypofractionated radiotherapy should only be used with radiotherapy alone or sequential CRT, whereas this should be avoided when large volumes are treated using concurrent CRT [7].

Toxicity can be reduced by limiting radiation fields to sites of known disease. For operable lung tumours, use of a systematic endobronchial ultrasound (EBUS) followed by an oesophageal investigation using the same EBUS-scope increases sensitivity for the detection of N2/N3 disease by $9 \%$ compared to use of positron emission tomography (PET)-CT targeted EBUS alone [91]. However, the role of systematic endoscopic nodal staging is unclear in patients undergoing CRT for inoperable, multi-level N2 disease. Similarly to treatment planning for early-stage disease, a respiration-correlated 4D-CT scan is desirable for radiotherapy planning in locally advanced NSCLC, as it allows for target and normal organ motion to be considered [4]. In addition, information from co-registered diagnostic PET-CT scans can improve target delineation, and the safety and efficacy of PET-based target definition has been demonstrated in a recent prospective study [92]. Limiting the target volume to the primary tumour and involved nodes, or suspected lymph node metastases, is referred to as involved-field radiotherapy. Involved-field radiotherapy represents the current standard of care in both European [4] and North American guidelines [93]. This underlines the importance of incorporating all information gathered from endoscopic nodal staging and imaging studies into the radiotherapy planning process. Use of larger, so-called "elective” nodal fields has been associated 
with worse survival [93], a finding which may be due to the risk of radiation-induced cardiac mortality [94] and immunosuppression [95].

In patients with a stage III NSCLC, CRT results in a 5-15\% likelihood of symptomatic radiation pneumonitis (table 1) [88, 96, 98, 101]. In the PACIFIC trial, any grade of (radiation) pneumonitis occurred in $33.9 \%$ and $24.8 \%$, with grade $3-4$ pneumonitis reported in $3.4 \%$ and $2.6 \%$ of the durvalumab and placebo groups, respectively [14, 15]. This emphasises the need for careful attention to identifying high-risk patients, for whom optimal radiotherapy delivery techniques [4] and supportive care during and after combined modality treatment are essential [102]. Optimising radiotherapy planning parameters, such as the mean lung dose or the lung volume receiving a certain threshold dose (e.g. $20 \mathrm{~Gy}$ ), can minimise the risk of radiation pneumonitis [4]. Doses to the heart and the oesophagus should be kept as low as possible, although no clear safe dose thresholds have been identified. Importantly, the introduction of new systemic treatments may potentially change the spectrum and frequency of adverse events observed during CRT (figure 4). Although the PACIFIC trial reported no significant increase in high-grade pneumonitis with durvalumab, a 14\% incidence of grade 3 radiation pneumonitis was reported in real-world data from a Korean centre, where more than half of the patients receiving durvalumab consolidation did not meet the inclusion criteria of the PACIFIC study [103]. When nivolumab, a programmed cell death protein 1 inhibitor, was administered during CRT in stage III NSCLC (NICOLAS trial), any grade pneumonitis was reported in $42.5 \%$ of patients, with $10.4 \%$ experiencing grade 3 pneumonitis [100]. Further improvements in radiation delivery, and improved patient selection, are areas of investigation in patients undergoing CRT with immune checkpoint inhibitors.

The severity of radiation-induced oesophagitis can be decreased by using appropriate dose fractionation schemes, and with IMRT techniques, which can allow for oesophagus-sparing delivery [104]. Limiting oesophagitis, and other acute toxicities, may also allow for the timely initiation of durvalumab consolidation, as an unplanned subset analysis of the PACIFIC trial suggested an improvement in progression-free survival (PFS) if durvalumab was initiated within 2 weeks after completion of CRT [14, 15].

Despite the advances in treatment of stage III NSCLC, improvements in population survival rates are less apparent for this subgroup of patients. Large variations exist in real-world treatment patterns for patients with stage III NSCLC, especially for the elderly. For example, only $11 \%$ of stage III NSCLC patients in England received chemotherapy and radiotherapy, with sequential CRT delivered almost twice as often as concurrent CRT. Furthermore, only 33\% of all patients with stage III disease, of whom one-third did not receive active treatment, survived for $>1$ year [105]. The complexity is further increased by a variability in expert opinions on best practices, particularly in N2 disease [106]. A population-based study in the Netherlands and Belgium showed that older age and higher N-stage were associated with the choice for sequential CRT [107]. A National Cancer Database (NCDB) study reported that sequential CRT was superior to concurrent CRT in elderly patients, suggesting a need for better tailoring of treatments in the elderly [108].

TABLE 1 Incidence of oesophagitis and pneumonitis observed in selected recent trials

\begin{tabular}{|c|c|c|c|c|}
\hline \multirow[t]{2}{*}{ Toxicity: CTCAE } & \multicolumn{2}{|c|}{ Pneumonitis \% } & \multicolumn{2}{|c|}{ Oesophagitis $\%$} \\
\hline & Any grade & Grade 3-4 & Any grade & Grade $3-4$ \\
\hline PROCLAIM: standard therapy [96] & 10.7 & 2.6 & 50.7 & 20.6 \\
\hline RTOG 0617: 60 Gy [97] & $\begin{array}{c}\text { Acute } 11.1 ; \\
\text { late } 9.8\end{array}$ & $\begin{array}{c}\text { Acute } 5.5 ; \\
\text { late } 1.2\end{array}$ & 45.1 & 6.9 \\
\hline RTOG 0617: 74 Gy [97] & $\begin{array}{c}\text { Acute } 14.5 ; \\
\text { late } 3.9\end{array}$ & $\begin{array}{c}\text { Acute } 3.4 ; \\
\text { late } 1.1\end{array}$ & 54.1 & 17 \\
\hline KCSG-LU05-04: CRT phase [98] & 2.4 & 0 & 79.3 & 9.5 \\
\hline CONVERT: LD-SCLC, once daily [99] & $\begin{array}{c}\text { Acute } 22.4 ; \\
\text { late } 32.6\end{array}$ & $\begin{array}{c}\text { Acute } 1.6 ; \\
\text { late } 2.6\end{array}$ & $\begin{array}{l}\text { Acute } 74 ; \\
\text { late } 18.5\end{array}$ & $\begin{array}{c}\text { Acute } 19.1 ; \\
\text { late } 1.7\end{array}$ \\
\hline CONVERT: LD-SCLC, twice daily [99] & $\begin{array}{c}\text { Acute } 22 ; \\
\text { late } 31\end{array}$ & $\begin{array}{c}\text { Acute } 1.6 \\
\text { late } 2.4\end{array}$ & $\begin{array}{c}\text { Acute } 81.1 ; \\
\text { late } 11.7\end{array}$ & $\begin{array}{c}\text { Acute } 18.5 ; \\
\text { late } 0\end{array}$ \\
\hline PACIFIC: durvalumab [15] & 33.9 & 3.4 & & \\
\hline PACIFIC: placebo [15] & 24.8 & 2.6 & & \\
\hline NICOLAS: nivolumab [100] & 42.5 & 10.4 & 27.5 & 6.5 \\
\hline
\end{tabular}



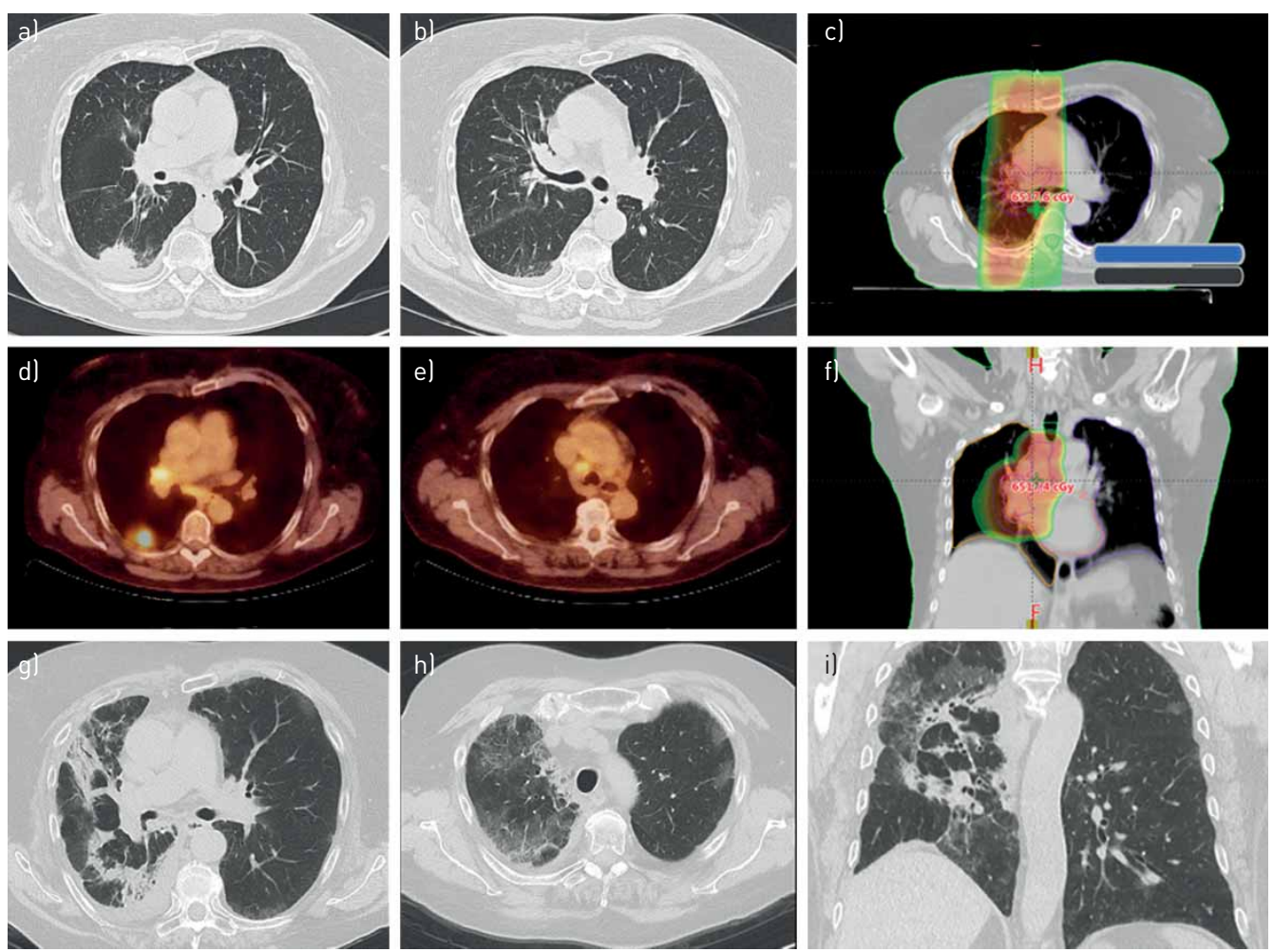

FIGURE 4 a, b, d, e) Serial images of a 70-year-old female patient with stage III nonsmall cell lung cancer who had completed chemoradiotherapy followed by durvalumab. c, f) The radiotherapy treatment plan with a colour-wash display of regions receiving a radiation dose of $30 \mathrm{~Gy}$ or higher. 2 months after commencing durvalumab the patient was diagnosed with a grade 2 radiation pneumonitis based upon diffuse ground-glass changes in the irradiated regions in the right lung. g-i) Subsequently, a diagnosis of coexisting immune checkpoint blockade pneumonitis was made based on the appearance of ground-glass abnormalities in the unirradiated contralateral upper lobe.

The growing use of improved radiation treatment planning and delivery techniques, such as IMRT and VMAT, has not been shown to adversely impact survival in population-based studies of stage III NSCLC, suggesting that use of these techniques should be encouraged due to their ability to spare normal organs [109]. Improved radiation delivery is also relevant because survivors remain at risk for developing second primary lung cancer, which occurs at a rate of $2.9 \%$ per patient per year after CRT [110]. Although proton delivery may potentially reduce low-dose exposure of uninvolved lung tissue, a prospective trial using Bayesian adaptive randomisation of proton therapy versus conventional IMRT did not show any improvements in the rates of radiation pneumonitis or local failure with proton therapy [111]. Ongoing studies, such as the RTOG 1308 trial, are using improved proton delivery approaches to explore the role of this approach in decreasing doses to organs at risk, such as the heart.

The role of post-operative radiotherapy (PORT) in patients with a resected stage III NSCLC had been controversial, as a meta-analysis in the late 1990s reported that PORT was detrimental to survival in patients with resected NSCLC pN0 and pN1 [112]. There had been uncertainty regarding the utility of PORT in patients with pN2 disease, especially as subsequent analyses from the SEER and NCDB databases had suggested that PORT could improve overall survival in patients with N2 disease [113-115]. LungART was a well-powered, prospective trial in this patient group which randomised 501 patients with completely resected stage IIIA N2 NSCLC to either PORT (54 Gy in 27-30 fractions) or no PORT [116]. The study reported no significant differences in the primary end-point of disease-free survival. In addition, the rates of grade 3-4 cardiopulmonary toxicity were more than doubled with PORT. It can therefore be concluded that PORT is not beneficial for most patients with a completely resected stage IIIA N2 NSCLC, and it is doubtful if improvements in radiotherapy delivery will be sufficient as to improve on these outcomes.

In the treatment of limited disease small cell lung cancer (SCLC), concurrent CRT is the standard of care, and radiotherapy planning principles are similar to those discussed for stage III NSCLC. Concurrent CRT 
with twice-daily radiotherapy to a dose of $45 \mathrm{~Gy}$ was established as a standard treatment based on a randomised trial that revealed superior outcomes compared to once-daily radiotherapy to 45 Gy in limited disease-SCLC [117]. However, not all centres perform twice-daily radiotherapy, as this can be a logistical challenge for departments and patients. The recent CONVERT trial compared twice-daily radiotherapy to $45 \mathrm{~Gy}$ as a standard of care, to once-daily radiotherapy to $66 \mathrm{~Gy}$, in a randomised superiority trial. No major differences were seen in survival or toxicity between twice-daily and once-daily radiotherapy [99]. Although twice-daily radiotherapy to 45 Gy is a current standard of care, a recent randomised phase II trial suggested that survival was superior with twice-daily thoracic radiotherapy to $60 \mathrm{~Gy}$, as opposed to 45 Gy [118]. However, the limitations of the phase II trial design mean that a confirmatory phase III trial must be performed to establish the superiority of a higher dose twice-daily radiotherapy scheme [119]. The ongoing ADRIATIC trial is evaluating the use of consolidation immunotherapy in patients with limited disease-SCLC who do not progress after concurrent CRT [120], although a similar study, STIMULI, recently reported no improvement of either PFS or overall survival with such an approach [121].

Chemotherapy is the treatment backbone for patients presenting with extensive disease SCLC, and two recent trials have revealed that the addition of immune checkpoint inhibitors to chemotherapy improves overall survival in these patients [122, 123]. The potential role of consolidative radiotherapy following first-line chemotherapy was previously investigated [124-126]. The CREST trial randomised patients with extensive disease-SCLC who responded to chemotherapy to either thoracic radiotherapy (30 Gy in 10 fractions) or no thoracic radiotherapy. CREST failed to meet its primary end-point of improved overall survival at 1 year, although thoracic radiotherapy increased 2-year overall survival rates from 3\% to $13 \%$ [124]. As the trials evaluating immunotherapy with first-line chemotherapy did not permit consolidative thoracic radiotherapy [122, 123], the role of thoracic radiotherapy in the era of chemoimmunotherapy is the subject of an ongoing study [127].

SABR for oligometastatic disease

SABR is increasingly used for the treatment of patients presenting with up to five metastases, or so-called oligometastatic disease. In patients with limited metastatic NSCLC who do not progress after first-line systemic therapy, local consolidative therapy can improve PFS compared to standard treatment [16, 17]. The randomised SABR-COMET trial demonstrated that SABR delivered to one to five metastatic lesions can improve long-term survival compared to standard of care in a mixed cohort of patients presenting with different primary tumours $[18,20]$. Nearly half of all metastases treated in the SABR-COMET trial were located in the lung, and lung SABR accounted for two of three fatal toxicities observed in the trial [18, 20]. Additional drivers of the need for improving SABR delivery are the suboptimal local control rates observed for some patients, with an overall long-term local control rate of only $63 \%$ for metastases in the SABR-COMET trial [20].

SABR has also been used for the treatment of isolated thoracic nodal recurrences [128, 129]. The results of ongoing trials, such as SABR-COMET-10 [78], may lead to a broadening of the indication for patients to undergo SABR for multiple lesions. Improved SABR delivery techniques will be required in such patients in order to reduce lung doses. Scheduling SABR treatments to multiple sites between cycles of systemic therapy will be challenging, and this may be facilitated by delivering shorter treatments. The safety, efficacy and cost-effectiveness of single-fraction SABR for oligometastatic patients with one to three lung metastases are being compared to a four-fraction SABR regimen in a randomised phase II trial, which has completed accrual [130].

Brain metastases are a major cause of morbidity and mortality in lung cancer. The management of these patients has become complex due to differences in prognosis based on factors such as the patient's performance status, number of brain metastases, extracranial disease and the presence of targetable (e.g. EGFR or ALK) gene alterations [131]. There is a role for whole brain radiotherapy (WBRT) to provide symptom relief in patients with brain metastases, although WBRT provides little additional benefit for quality of life or survival in patients with brain metastases from NSCLC who are unsuitable for surgical resection or stereotactic radiotherapy [132]. In contrast to WBRT, single-session radiosurgery has become established as an effective local treatment for patients with up to 10 brain metastases [133, 134]. Furthermore, as modern magnetic resonance imaging allows for the detection of very small brain metastases, regular imaging allows for repeated stereotactic irradiation of new lesions, a concept which is currently being evaluated in a prospective trial [135]. For patients with SCLC, administration of prophylactic cranial irradiation following chemoradiation in limited disease-SCLC is considered standard due to the high risk of central nervous system (CNS) relapse [136]. Due to the risk of disseminated CNS progression, WBRT has traditionally been recommended in patients with brain metastases from SCLC, although this paradigm has been challenged by a recent cohort study suggesting that similar overall survival outcomes can be achieved with first-line 
radiosurgery [137]. First-line brain radiosurgery for patients with SCLC and up to 10 brain metastases is now being evaluated in a prospective randomised trial [138].

In a broader context, the role of radiation therapy in metastatic disease is being transformed by rapid changes in systemic treatments. In particular, the synergy of radiotherapy and immunotherapy remains an active area of research [139]. Both preclinical and clinical studies are aiming to better understand the complex immunomodulatory effects of radiation, and how to best use its potential to induce a systemic antitumour immune response [48]. In a prospective clinical trial in patients with progressive NSCLC where SABR was delivered to a single metastatic lesion prior to pembrolizumab ( $200 \mathrm{mg} \cdot \mathrm{kg}^{-1}$ every 3 weeks) versus pembrolizumab alone, a doubling of overall response rates was observed, although the results did not meet the study’s prespecified end-point criteria for meaningful clinical benefit [19].

\section{Conclusion}

Recent advances in radiation therapy have contributed to the improvements observed in the survival of patients presenting with a lung cancer. Clear examples of this are the decreasing rates of non-treatment in early-stage lung cancer in population studies and the survival gains observed in trials incorporating immunotherapy following CRT in locally advanced NSCLC. In many parts of the world, the lack of patient access to these newer techniques remains an impediment. Similarly, as treatment options become more complex, such as for oligometastatic lung cancer, the role of the multidisciplinary tumour board in selecting appropriate strategies will be paramount.

Provenance: Commissioned article, peer reviewed.

Previous articles in this series: No. 1: Eichhorn F, Winter H. How to handle oligometastatic disease in nonsmall cell lung cancer. Eur Respir Rev 2021; 30: 200234. No. 2: Asciak R, George V, Rahmna NM. Update on biology and management of mesothelioma. Eur Respir Rev 2021; 30: 200226.

Conflict of interest: T. Finazzi reports personal fees from AstraZeneca, outside the submitted work. F.L. Schneiders has nothing to disclose. S. Senan reports grants and personal fees from AstraZeneca and Varian Medical Systems, grants from ViewRay Inc., and personal fees from MSD and Celgene, outside the submitted work.

\section{References}

1 Sung H, Ferlay J, Siegel RL, et al. Global cancer statistics 2020: GLOBOCAN estimates of incidence and mortality worldwide for 36 cancers in 185 countries. CA Cancer J Clin 2021; in press [https://doi.org/10.3322/ caac.21660].

2 Guckenberger M, Andratschke N, Dieckmann K, et al. ESTRO ACROP consensus guideline on implementation and practice of stereotactic body radiotherapy for peripherally located early stage non-small cell lung cancer. Radiother Oncol 2017; 124: 11-17.

3 Videtic GMM, Donington J, Giuliani M, et al. Stereotactic body radiation therapy for early-stage non-small cell lung cancer: executive summary of an ASTRO evidence-based guideline. Pract Radiat Oncol 2017; 7: 295-301.

4 De Ruysscher D, Faivre-Finn C, Moeller D, et al. European Organization for Research and Treatment of Cancer (EORTC) recommendations for planning and delivery of high-dose, high precision radiotherapy for lung cancer. Radiother Oncol 2017; 124: 1-10.

5 Postmus PE, Kerr KM, Oudkerk M, et al. Early and locally advanced non-small-cell lung cancer (NSCLC): ESMO Clinical Practice Guidelines for diagnosis, treatment and follow-up. Ann Oncol 2017; 28: iv1-iv21.

6 National Comprehensive Cancer Network. NCCN Guidelines version 4.2021: Non-Small Cell Lung Cancer. www.nccn.org/professionals/physician_gls/pdf/nscl.pdf Date last updated: 3 March 2021. Date last accessed: 18 April 2021.

7 Guckenberger M, Belka C, Bezjak A, et al. Practice recommendations for lung cancer radiotherapy during the COVID-19 pandemic: An ESTRO-ASTRO consensus statement. Radiother Oncol 2020; 146: 223-229.

8 Nanda RH, Liu Y, Gillespie TW, et al. Stereotactic body radiation therapy versus no treatment for early stage non-small cell lung cancer in medically inoperable elderly patients: a National Cancer Data Base analysis. Cancer 2015; 121: 4222-4230.

9 Haasbeek CJA, Palma D, Visser O, et al. Early-stage lung cancer in elderly patients: a population-based study of changes in treatment patterns and survival in the Netherlands. Ann Oncol 2012; 23: 2743-2747.

10 Chen AB, Neville BA, Sher DJ, et al. Survival outcomes after radiation therapy for stage III non-small-cell lung cancer after adoption of computed tomography-based simulation. J Clin Oncol 2011; 29: 2305-2311.

11 Jegadeesh N, Liu Y, Gillespie T, et al. Evaluating intensity-modulated radiation therapy in locally advanced non-small-cell lung cancer: results from the national cancer data base. Clin Lung Cancer 2016; 17: 398-405. 
Phillips I, Sandhu S, Lüchtenborg M, et al. Stereotactic ablative body radiotherapy versus radical radiotherapy: comparing real-world outcomes in stage I lung cancer. Clin Oncol (R Coll Radiol) 2019; 31: 681-687.

Ball D, Mai GT, Vinod S, et al. Stereotactic ablative radiotherapy versus standard radiotherapy in stage 1 non-small-cell lung cancer (TROG 09.02 CHISEL): a phase 3, open-label, randomised controlled trial. Lancet Oncol 2019; 20: 494-503.

Antonia SJ, Villegas A, Daniel D, et al. Overall survival with durvalumab after chemoradiotherapy in stage III NSCLC. N Engl J Med 2018; 379: 2342-2350.

Antonia SJ, Villegas A, Daniel D, et al. Durvalumab after chemoradiotherapy in stage III non-small-cell lung cancer. N Engl J Med 2017; 377: 1919-1929.

Gomez DR, Blumenschein GR, Lee JJ, et al. Local consolidative therapy versus maintenance therapy or observation for patients with oligometastatic non-small-cell lung cancer without progression after first-line systemic therapy: a multicentre, randomised, controlled, phase 2 study. Lancet Oncol 2016; 17: 1672-1682.

lyengar P, Wardak Z, Gerber DE, et al. Consolidative radiotherapy for limited metastatic non-small-cell lung cancer: a phase 2 randomized clinical trial. JAMA Oncol 2018; 4: e173501.

Palma DA, Olson R, Harrow S, et al. Stereotactic ablative radiotherapy versus standard of care palliative treatment in patients with oligometastatic cancers (SABR-COMET): a randomised, phase 2, open-label trial. Lancet 2019; 393: 2051-2058.

Theelen WSME, Peulen HMU, Lalezari F, et al. Effect of pembrolizumab after stereotactic body radiotherapy vs pembrolizumab alone on tumor response in patients with advanced non-small cell lung cancer. JAMA Oncol 2019; 5: 1276-1282.

Palma D, Olson R, Harrow S, et al. Stereotactic ablative radiotherapy for the comprehensive treatment of oligometastatic cancers: long-term results of the SABR-COMET phase II randomized trial. J Clin Oncol 2020; 38: 2830-2838.

Blomgren H, Lax I, Näslund I, et al. Stereotactic high dose fraction radiation therapy of extracranial tumors using an accelerator. Clinical experience of the first thirty-one patients. Acta Oncol 1995; 34: 861-870.

Nyman J, Hallqvist A, Lund JÅ, et al. SPACE - a randomized study of SBRT vs conventional fractionated radiotherapy in medically inoperable stage I NSCLC. Radiother Oncol 2016; 121: 1-8.

Videtic GM, Paulus R, Singh AK, et al. Long-term follow-up on NRG oncology RTOG 0915 (NCCTG N0927): a randomized phase 2 study comparing 2 stereotactic body radiation therapy schedules for medically inoperable patients with stage I peripheral non-small cell lung cancer. Int J Radiat Oncol Biol Phys 2019; 103: 1077-1084.

Singh AK, Gomez-Suescun JA, Stephans KL, et al. One versus three fractions of stereotactic body radiation therapy for peripheral stage I-II non-small cell lung cancer: a randomized, multi-institution, phase 2 trial. Int J Radiat Oncol Biol Phys 2019; 105: 752-759.

Soldà F, Lodge M, Ashley S, et al. Stereotactic radiotherapy (SABR) for the treatment of primary non-small cell lung cancer: systematic review and comparison with a surgical cohort. Radiother Oncol 2013; 109: 1-7.

Grills IS, Mangona VS, Welsh R, et al. Outcomes after stereotactic lung radiotherapy or wedge resection for stage I non-small-cell lung cancer. J Clin Oncol 2010; 28: 928-935.

Bryant AK, Mundt RC, Sandhu AP, et al. Stereotactic body radiation therapy versus surgery for early lung cancer among US veterans. Ann Thorac Surg 2017; 105: 425-431.

Ronden MI, Palma D, Slotman BJ, et al. Brief report on radiological changes following stereotactic ablative radiotherapy (SABR) for early-stage lung tumors: a pictorial essay. J Thorac Oncol 2018; 13: 855-862.

Dahele M, Palma D, Lagerwaard F, et al. Radiological changes after stereotactic radiotherapy for stage I lung cancer. J Thorac Oncol 2011; 6: 1221-1228.

Brooks ED, Verma V, Senan S, et al. Salvage therapy for locoregional recurrence after stereotactic ablative radiotherapy for early-stage non-small cell lung cancer. J Thorac Oncol 2020; 15: 176-189.

Chang JY, Senan S, Paul MA, et al. Stereotactic ablative radiotherapy versus lobectomy for operable stage I non-small-cell lung cancer: a pooled analysis of two randomised trials. Lancet Oncol 2015; 16: 630-637.

Subramanian MP, Meyers BF. Surgical resection versus stereotactic body radiation therapy for stage I NSCLC: can randomized trials provide the solution? Cancers (Basel) 2018; 10: 310.

de Ruiter JC, Heineman DJ, Daniels JM, et al. The role of surgery for stage I non-small cell lung cancer in octogenarians in the era of stereotactic body radiotherapy in the Netherlands. Lung Cancer 2020; 144: 64-70.

Roach MC, Robinson CG, DeWees TA, et al. Stereotactic body radiation therapy for central early-stage NSCLC: results of a prospective phase I/II trial. J Thorac Oncol 2018; 13: 1727-1732.

Tekatli H, Haasbeek N, Dahele M, et al. Outcomes of hypofractionated high-dose radiotherapy in poor-risk patients with "ultracentral" non-small cell lung cancer. J Thorac Oncol 2016; 11: 1081-1089.

36 Haseltine JM, Rimner A, Gelblum DY, et al. Fatal complications after stereotactic body radiation therapy for central lung tumors abutting the proximal bronchial tree. Pract Radiat Oncol 2016; 6: e27-e33.

Timmerman R, McGarry R, Yiannoutsos C, et al. Excessive toxicity when treating central tumors in a phase II study of stereotactic body radiation therapy for medically inoperable early-stage lung cancer. $J$ Clin Oncol 2006; 24: 4833-4839. 
Tekatli H, van 't Hof S, Nossent EJ, et al. Use of stereotactic ablative radiotherapy (SABR) in non-small cell lung cancer measuring more than $5 \mathrm{~cm}$. J Thorac Oncol 2017; 12: 974-982.

Chen $\mathrm{H}$, Senan S, Nossent EJ, et al. Treatment-related toxicity in patients with early-stage non-small cell lung cancer and coexisting interstitial lung disease: a systematic review. Int J Radiat Oncol Biol Phys 2017; 98: 622-631.

Finazzi T, Ronden-Kianoush MI, Spoelstra FOB, et al. Stereotactic ablative radiotherapy in patients with early-stage non-small cell lung cancer and co-existing interstitial lung disease. Acta Oncol 2020; 59: 569-573.

Sun B, Brooks ED, Komaki R, et al. Long-term outcomes of salvage stereotactic ablative radiotherapy for isolated lung recurrence of non-small cell lung cancer: a phase II clinical trial. J Thorac Oncol 2017; 12: 983-992.

Hamaji M, Ali SO, Burt BM. A meta-analysis of resected metachronous second non-small cell lung cancer. Ann Thorac Surg 2015; 99: 1470-1478.

Leroy T, Monnet E, Guerzider S, et al. Let us not underestimate the long-term risk of SPLC after surgical resection of NSCLC. Lung Cancer 2019; 137: 23-30.

Senthi S, Lagerwaard FJ, Haasbeek CJA, et al. Patterns of disease recurrence after stereotactic ablative radiotherapy for early stage non-small-cell lung cancer: a retrospective analysis. Lancet Oncol 2012; 13: 802-809.

Chang JY, Lin SH, Yao L, et al. I-SABR phase II randomized study of nivolumab immunotherapy and stereotactic ablative radiotherapy in early stage NSCLC: interim analysis adverse effects. J Clin Oncol 2020; 38: Suppl. 15, 9035.

ClinicalTrials.gov. Durvalumab vs Placebo With Stereotactic Body Radiation Therapy in Early Stage Unresected Non-small Cell Lung Cancer Patients. NCT03833154. https://clinicaltrials.gov/ct2/show/ NCT03833154 Date last updated: 22 January 2021. Date last accessed: 21 September 2020.

ClinicalTrials.gov. Efficacy and Safety Study of Stereotactic Body Radiotherapy (SBRT) with or without Pembrolizumab (MK-3475) in Adults With Medically Inoperable Stage I or IIA Non-Small Cell Lung Cancer (NSCLC) (MK-3475-867/KEYNOTE-867). NCT03924869. https://clinicaltrials.gov/ct2/show/NCT03924869 Date last updated: 25 January 2021. Date last accessed: 21 September 2020.

Marciscano AE, Haimovitz-Friedman A, Lee P, et al. Immunomodulatory effects of stereotactic body radiation therapy: preclinical insights and clinical opportunities. Int J Radiat Oncol Biol Phys 2021; 110: 35-52.

Grills IS, Yan D, Martinez AA, et al. Potential for reduced toxicity and dose escalation in the treatment of inoperable non-small-cell lung cancer: a comparison of intensity-modulated radiation therapy (IMRT), 3D conformal radiation, and elective nodal irradiation. Int J Radiat Oncol Biol Phys 2003; 57: 875-890.

Chapet $\mathrm{O}$, Khodri M, Jalade P, et al. Potential benefits of using non coplanar field and intensity modulated radiation therapy to preserve the heart in irradiation of lung tumors in the middle and lower lobes. Radiother Oncol 2006; 80: 333-340.

Murshed H, Liu HH, Liao Z, et al. Dose and volume reduction for normal lung using intensity-modulated radiotherapy for advanced-stage non-small-cell lung cancer. Int J Radiat Oncol Biol Phys 2004; 58: 1258-1267.

De Ruysscher D, Sterpin E, Haustermans K, et al. Tumour movement in proton therapy: solutions and remaining questions: a review. Cancers (Basel) 2015; 7: 1143-1153.

Chang JY, Jabbour SK, De Ruysscher D, et al. Consensus statement on proton therapy in early-stage and locally advanced non-small cell lung cancer. Int J Radiat Oncol Biol Phys 2016; 95: 505-516.

Seco J, Panahandeh HR, Westover K, et al. Treatment of non-small cell lung cancer patients with proton beam-based stereotactic body radiotherapy: dosimetric comparison with photon plans highlights importance of range uncertainty. Int J Radiat Oncol Biol Phys 2012; 83: 354-361.

Westover KD, Seco J, Adams JA, et al. Proton SBRT for medically inoperable stage I NSCLC. J Thorac Oncol 2012; 7: 1021-1025.

Grant JD, Chang JY. Proton-based stereotactic ablative radiotherapy in early-stage non-small-cell lung cancer. Biomed Res Int 2014; 2014: 389048.

Ehrbar S, Jöhl A, Tartas A, et al. ITV, mid-ventilation, gating or couch tracking - a comparison of respiratory motion-management techniques based on 4D dose calculations. Radiother Oncol 2017; 124: 80-88.

Steiner E, Shieh CC, Caillet V, et al. Both four-dimensional computed tomography and four-dimensional cone beam computed tomography under-predict lung target motion during radiotherapy. Radiother Oncol 2019; 135: 65-73.

Cusumano D, Dhont J, Boldrini L, et al. Predicting tumour motion during the whole radiotherapy treatment: a systematic approach for thoracic and abdominal lesions based on real time MR. Radiother Oncol 2018; 129: 456-462.

Finazzi T, Palacios MA, Haasbeek CJA, et al. Stereotactic MR-guided adaptive radiation therapy for peripheral lung tumors. Radiother Oncol 2020; 144: 46-52.

Underberg RWM, Lagerwaard FJ, Slotman BJ, et al. Benefit of respiration-gated stereotactic radiotherapy for stage I lung cancer: an analysis of 4DCT datasets. Int J Radiat Oncol Biol Phys 2005; 62: 554-560. 
Jang SS, Huh GJ, Park SY, et al. The impact of respiratory gating on lung dosimetry in stereotactic body radiotherapy for lung cancer. Phys Med 2014; 30: 682-689.

Marchand V, Zefkili S, Desrousseaux J, et al. Dosimetric comparison of free-breathing and deep inspiration breath-hold radiotherapy for lung cancer. Strahlenther Onkol 2012; 188: 582-589.

Whyte RI, Crownover R, Murphy MJ, et al. Stereotactic radiosurgery for lung tumors: Preliminary report of a phase I trial. Ann Thorac Surg 2003; 75: 1097-1101.

Schmitt D, Nill S, Roeder F, et al. Motion monitoring during a course of lung radiotherapy with anchored electromagnetic transponders: quantification of inter- and intrafraction motion and variability of relative transponder positions. Strahlenther Onkol 2017; 193: 840-847.

Scher N, Bollet M, Bouilhol G, et al. Safety and efficacy of fiducial marker implantation for robotic SBRT with fiducial tracking. Radiat Oncol 2019; 14: 167.

Bhagat N, Fidelman N, Durack JC, et al. Complications associated with the percutaneous insertion of fiducial markers in the thorax. Cardiovasc Intervent Radiol 2010; 33: 1186-1191.

Giraud P, Morvan E, Claude L, et al. Respiratory gating techniques for optimization of lung cancer radiotherapy. J Thorac Oncol 2011; 6: 2058-2068.

Heinzerling $\mathrm{JH}$, Hampton $\mathrm{CJ}$, Robinson $\mathrm{M}$, et al. Use of surface-guided radiation therapy in combination with IGRT for setup and intrafraction motion monitoring during stereotactic body radiation therapy treatments of the lung and abdomen. J Appl Clin Med Phys 2020; 21: 48-55.

Park S, Farah R, Shea SM, et al. Simultaneous tumor and surrogate motion tracking with dynamic MRI for radiation therapy planning. Phys Med Biol 2019; 63: 1-24.

Winkel D, Bol GH, Kroon PS, et al. Adaptive radiotherapy: The Elekta Unity MR-linac concept. Clin Trans Radiat Oncol 2019; 18: 54-59.

Mutic S, Dempsey JF. The ViewRay System: magnetic resonance-guided and controlled radiotherapy. Semin Radiat Oncol 2014; 24: 196-199.

van Sörnsen de Koste JR, Palacios MA, Bruynzeel AME, et al. MR-guided gated stereotactic radiation therapy delivery for lung, adrenal, and pancreatic tumors: a geometric analysis. Int J Radiat Oncol Biol Phys 2018; 102: 858-866.

Finazzi T, Palacios MA, Spoelstra FOB, et al. Role of on-table plan adaptation in MR-guided ablative radiation therapy for central lung tumors. Int J Radiat Oncol Biol Phys 2019; 104: 933-941.

Henke LE, Olsen JR, Contreras JA, et al. Stereotactic MR-guided online adaptive radiation therapy (SMART) for ultracentral thorax malignancies: results of a phase I trial. Adv Radiat Oncol 2018; 4: 201-209.

Henke L, Kashani R, Yang D, et al. Simulated online adaptive magnetic resonance-guided stereotactic body radiation therapy for the treatment of oligometastatic disease of the abdomen and central thorax: characterization of potential advantages. Int J Radiat Oncol Biol Phys 2016; 96: 1078-1086.

Henke LE, Kashani R, Hilliard J, et al. In silico trial of MR-guided midtreatment adaptive planning for hypofractionated stereotactic radiation therapy in centrally located thoracic tumors. Int J Radiat Oncol Biol Phys 2018; 102: 987-995.

Palma DA, Olson R, Harrow S, et al. Stereotactic ablative radiotherapy for the comprehensive treatment of 4-10 oligometastatic tumors (SABR-COMET-10): study protocol for a randomized phase III trial. BMC Cancer 2019; 19: 816.

Finazzi T, De Koste JRVS, Palacios MA, et al. Delivery of magnetic resonance-guided single-fraction stereotactic lung radiotherapy. Phys Imaging Radiat Oncol 2020; 14: 17-23.

Finazzi T, Haasbeek CJA, Spoelstra FOB, et al. Clinical outcomes of stereotactic MR-guided adaptive radiation therapy for high-risk lung tumors. Int J Radiat Oncol Biol Phys 2020; 107: 270-278.

Kumar S, Liney G, Rai R, et al. Magnetic resonance imaging in lung: a review of its potential for radiotherapy. Br J Radiol 2016; 89: 20150431.

Eberhardt WEE, De Ruysscher D, Weder W, et al. 2nd ESMO Consensus Conference in Lung Cancer: locally advanced stage III non-small-cell lung cancer. Ann Oncol 2015; 26: 1573-1588.

Kris MG, Faivre-Finn C, Kordbacheh T, et al. Making checkpoint inhibitors part of treatment of patients with locally advanced lung cancers: the time is now. Am Soc Clin Oncol Educ Book 2020; 40: 1-12.

Peters S, Weder W, Dafni U, et al. Lungscape: Resected non-small-cell lung cancer outcome by clinical and pathological parameters. J Thorac Oncol 2014; 9: 1675-1684.

Park K, Vansteenkiste J, Lee KH, et al. Pan-Asian adapted ESMO Clinical Practice Guidelines for the management of patients with locally-advanced unresectable non-small-cell lung cancer: a KSMO-ESMO initiative endorsed by CSCO, ISMPO, JSMO, MOS, SSO and TOS. Ann Oncol 2020; 31: 191-201.

86 Aupérin A, Le Péchoux C, Rolland E, et al. Meta-analysis of concomitant versus sequential radiochemotherapy in locally advanced non-small-cell lung cancer. J Clin Oncol 2010; 28: 2181-2190.

Faivre-Finn C, Vicente D, Kurata T, et al. Durvalumab after chemoradiotherapy in stage III NSCLC: 4-year survival update from the phase III PACIFIC trial. Ann Oncol 2020; 31: S1178-S1179. 

high-dose chemoradiotherapy with or without cetuximab for unresectable stage III non-small-cell lung cancer. J Clin Oncol 2020; 38: 706-714.

89 Maguire J, Khan I, McMenemin R, et al. SOCCAR: A randomised phase II trial comparing sequential versus concurrent chemotherapy and radical hypofractionated radiotherapy in patients with inoperable stage III non-small cell lung cancer and good performance status. Eur J Cancer 2014; 50: 2939-2949.

90 Passaro A, Addeo A, Von Garnier C, et al. ESMO Management and treatment adapted recommendations in the COVID-19 era: lung cancer. ESMO Open 2020; 5: e000820.

91 Crombag LMM, Dooms C, Stigt JA, et al. Systematic and combined endosonographic staging of lung cancer (SCORE study). Eur Respir J 2019; 53: 1800800.

92 Nestle U, Schimek-Jasch T, Kremp S, et al. Imaging-based target volume reduction in chemoradiotherapy for locally advanced non-small-cell lung cancer (PET-Plan): a multicentre, open-label, randomised, controlled trial. Lancet Oncol 2020; 21: 581-592.

93 Schild SE, Pang HH, Fan W, et al. Exploring radiotherapy targeting strategy and dose: a pooled analysis of cooperative group trials of combined modality therapy for stage III NSCLC. J Thorac Oncol 2018; 13: 1171-1182.

94 Atkins KM, Rawal B, Chaunzwa TL, et al. Cardiac radiation dose, cardiac disease, and mortality in patients with lung cancer. J Am Coll Cardiol 2019; 73: 2976-2987.

95 Contreras JA, Lin AJ, Weiner A, et al. Cardiac dose is associated with immunosuppression and poor survival in locally advanced non-small cell lung cancer. Radiother Oncol 2018; 128: 498-504.

96 Senan S, Brade A, Wang LH, et al. PROCLAIM: randomized phase III trial of pemetrexed-cisplatin or etoposide-cisplatin plus thoracic radiation therapy followed by consolidation chemotherapy in locally advanced nonsquamous non-small-cell lung cancer. J Clin Oncol 2016; 34: 953-962.

Bradley JD, Paulus R, Komaki R, et al. Standard-dose versus high-dose conformal radiotherapy with concurrent and consolidation carboplatin plus paclitaxel with or without cetuximab for patients with stage IIIA or IIIB non-small-cell lung cancer (RTOG 0617): a randomised, two-by-two factorial phase 3 study. Lancet Oncol 2015; 16: 187-199.

98 Ahn JS, Ahn YC, Kim J-H, et al. Multinational randomized phase III trial with or without consolidation chemotherapy using docetaxel and cisplatin after concurrent chemoradiation in inoperable stage III non-small-cell lung cancer: KCSG-LU05-04. J Clin Oncol 2015; 33: 2660-2666.

99 Faivre-Finn C, Snee M, Ashcroft L, et al. Concurrent once-daily versus twice-daily chemoradiotherapy in patients with limited-stage small-cell lung cancer (CONVERT): an open-label, phase 3, randomised, superiority trial. Lancet Oncol 2017; 18: 1116-1125.

100 Peters S, Felip E, Dafni U, et al. Safety evaluation of nivolumab added concurrently to radiotherapy in a standard first line chemo-radiotherapy regimen in stage III non-small cell lung cancer: The ETOP NICOLAS trial. Lung Cancer 2019; 133: 83-87.

101 Palma DA, Senan S, Tsujino K, et al. Predicting radiation pneumonitis after chemoradiation therapy for lung cancer: an international individual patient data meta-analysis. Int J Radiat Oncol Biol Phys 2013; 85: 444-450.

102 De Ruysscher D, Faivre-Finn C, Nackaerts K, et al. Recommendation for supportive care in patients receiving concurrent chemotherapy and radiotherapy for lung cancer. Ann Oncol 2020; 31: 41-49.

103 Jung HA, Noh JM, Sun J-M, et al. Real world data of durvalumab consolidation after chemoradiotherapy in stage III non-small-cell lung cancer. Lung Cancer 2020; 146: 23-29.

104 Ma L, Qiu B, Li Q, et al. An esophagus-sparing technique to limit radiation esophagitis in locally advanced non-small cell lung cancer treated by simultaneous integrated boost intensity-modulated radiotherapy and concurrent chemotherapy. Radiat Oncol 2018; 13: 130.

105 Adizie JB, Khakwani A, Beckett P, et al. Stage III non-small cell lung cancer management in England. Clin Oncol 2019; 31: 688-696.

106 Evison M, Edwards J, McDonald F, et al. Stage III non-small cell lung cancer: a UK national survey of practice. Clin Oncol 2020; 32: 527-536.

107 Walraven I, Damhuis RA, Ten Berge MG, et al. Treatment variation of sequential versus concurrent chemoradiotherapy in stage III non-small cell lung cancer patients in the Netherlands and Belgium. Clin Oncol 2017; 29: e177-e185.

108 Miller ED, Fisher JL, Haglund KE, et al. The addition of chemotherapy to radiation therapy improves survival in elderly patients with stage III non-small cell lung cancer. J Thorac Oncol 2018; 13: 426-435.

109 Peng J, Pond G, Donovan E, et al. A comparison of radiation techniques in patients treated with concurrent chemoradiation for Stage III non-small cell lung cancer. Int J Radiat Oncol Biol Phys 2020; 106: 985-992.

110 Kawaguchi T, Matsumura A, luchi K, et al. Second primary cancers in patients with stage III non-small cell lung cancer successfully treated with chemo-radiotherapy. Jpn J Clin Oncol 2006; 36: 7-11.

111 Liao Z, Lee JJ, Komaki R, et al. Bayesian adaptive randomization trial of passive scattering proton therapy and intensity-modulated photon radiotherapy for locally advanced non-small-cell lung cancer. J Clin Oncol 2018; 36: 1813-1822. 
112 PORT Meta-analysis Trialists Group. Postoperative radiotherapy in non-small-cell lung cancer: systematic review and meta-analysis of individual patient data from nine randomised controlled trials. Lancet 1998; 352: 257-263.

113 Herskovic A, Mauer E, Christos P, et al. Role of postoperative radiotherapy in pathologic stage IIIA (N2) non-small cell lung cancer in a prospective nationwide oncology outcomes database. $J$ Thorac Oncol 2017; 12: 302-313.

114 Lally BE. Postoperative radiotherapy for stage II or III non-small-cell lung cancer using the surveillance, epidemiology, and end results database. J Clin Oncol 2006; 24: 2998-3006.

115 Robinson CG, Patel AP, Bradley JD, et al. Postoperative radiotherapy for pathologic N2 non-small-cell lung cancer treated with adjuvant chemotherapy: a review of the national cancer data base. J Clin Oncol 2015; 33: 870-876.

116 Le Pechoux C, Pourel N, Barlesi F, et al. An international randomized trial, comparing post-operative conformal radiotherapy (PORT) to no PORT, in patients with completely resected non-small cell lung cancer (NSCLC) and mediastinal N2 involvement: primary end-point analysis of LungART (IFCT-0503). Ann Oncol 2020; 31: S1178.

117 Turrisi AT, Kim K, Blum R, et al. Twice-daily compared with once-daily thoracic radiotherapy in limited small-cell lung cancer treated concurrently with cisplatin and etoposide. N Engl J Med 1999; 340: 265-271.

118 Grønberg BH, Killingberg KT, Fløtten $\varnothing$, et al. High-dose versus standard-dose twice-daily thoracic radiotherapy for patients with limited stage small-cell lung cancer: an open-label, randomised, phase 2 trial. Lancet Oncol 2021; 22: 321-331.

119 Senan S. The elusive pursuit of progress in limited stage small-cell lung cancer. Lancet Oncol 2021; 22: 290-291.

120 Senan S, Okamoto I, Lee G-W, et al. Design and rationale for a phase III, randomized, placebo-controlled trial of durvalumab with or without tremelimumab after concurrent chemoradiotherapy for patients with limited-stage small-cell lung cancer: The ADRIATIC Study. Clin Lung Cancer 2020; 21: e84-e88.

121 Peters S, Pujol J-L, Dafni U, et al. Consolidation ipilimumab and nivolumab vs observation in limited stage SCLC after chemo-radiotherapy: results from the ETOP/IFCT 4-12 STIMULI trial. Ann Oncol 2020; 31: S1211.

122 Paz-Ares L, Dvorkin M, Chen Y, et al. Durvalumab plus platinum-etoposide versus platinum-etoposide in first-line treatment of extensive-stage small-cell lung cancer (CASPIAN): a randomised, controlled, open-label, phase 3 trial. Lancet 2019; 394: 1929-1939.

123 Horn L, Mansfield AS, Szczęsna A, et al. First-line atezolizumab plus chemotherapy in extensive-stage small-cell lung cancer. N Engl J Med 2018; 379: 2220-2229.

124 Slotman BJ, Van Tinteren H, Praag JO, et al. Use of thoracic radiotherapy for extensive stage small-cell lung cancer: a phase 3 randomised controlled trial. Lancet 2015; 385: 36-42.

125 Gore EM, Hu C, Sun AY, et al. Randomized phase II study comparing prophylactic cranial irradiation alone to prophylactic cranial irradiation and consolidative extracranial irradiation for extensive-disease small cell lung cancer (ED SCLC): NRG Oncology RTOG 0937. J Thorac Oncol 2017; 12: 1561-1570.

126 Jeremic BB, Shibamoto Y, Nikolic N, et al. Role of radiation therapy in the combined-modality treatment of patients with extensive disease small-cell lung cancer: a randomized study. J Clin Oncol 1999; 17: 2092-2099.

127 ClinicalTrials.gov. Thoracic RadiothErapy With Atezolizumab in Small Cell IUng canceR Extensive Disease (TREASURE). NCT04462276. https://clinicaltrials.gov/ct2/show/NCT04462276 Date last updated: 20 August 2020. Date last accessed: 21 September 2020.

128 Franceschini D, Bianciardi F, Mazzola R, et al. Can thoracic nodes oligometastases be safely treated with image guided hypofractionated radiation therapy? Br J Radiol 2019; 92: 20181026.

129 Horne ZD, Richman AH, Dohopolski MJ, et al. Stereotactic body radiation therapy for isolated hilar and mediastinal non-small cell lung cancers. Lung Cancer 2018; 115: 1-4.

130 Siva S, Kron T, Bressel M, et al. A randomised phase II trial of stereotactic ablative fractionated radiotherapy versus radiosurgery for oligometastatic neoplasia to the lung (TROG 13.01 SAFRON II). BMC Cancer 2016; 16: 1-8.

131 Sperduto PW, Mesko S, Li J, et al. Survival in patients with brain metastases: summary report on the updated diagnosis-specific graded prognostic assessment and definition of the eligibility quotient. $J$ Clin Oncol 2020; 38: 1-13.

132 Mulvenna P, Nankivell M, Barton R, et al. Dexamethasone and supportive care with or without whole brain radiotherapy in treating patients with non-small cell lung cancer with brain metastases unsuitable for resection or stereotactic radiotherapy (QUARTZ): results from a phase 3, non-inferiority, randomised trial. Lancet 2016; 388: 2004-2014.

133 Yamamoto M, Serizawa T, Shuto T, et al. Stereotactic radiosurgery for patients with multiple brain metastases (JLGK0901): a multi-institutional prospective observational study. Lancet Oncol 2014; 15: 387-395.

134 Brown PD, Jaeckle K, Ballman KV, et al. Effect of radiosurgery alone vs radiosurgery with whole brain radiation therapy on cognitive function in patients with 1 to 3 brain metastases. JAMA 2016; 316: 401-409.

135 El Shafie RA, Paul A, Bernhardt D, et al. Robotic radiosurgery for brain metastases diagnosed with either SPACE or MPRAGE sequence (CYBER-SPACE): a single-center prospective randomized trial. Neurosurgery 2019; 84: 253-260. 
136 Aupérin A, Arriagada R, Pignon JP, et al. Prophylactic cranial irradiation for patients with small-cell lung cancer in complete remission. N Engl J Med 1999; 341: 476-484.

137 Rusthoven CG, Yamamoto M, Bernhardt D, et al. Evaluation of first-line radiosurgery vs whole-brain radiotherapy for small cell lung cancer brain metastases: the FIRE-SCLC Cohort Study. JAMA Oncol 2020; 6: 1028-1037.

138 Bernhardt D, Hommertgen A, Schmitt D, et al. Whole brain radiation therapy alone versus radiosurgery for patients with 1-10 brain metastases from small cell lung cancer (ENCEPHALON Trial): study protocol for a randomized controlled trial. Trials 2018; 19: 1-8.

139 Walker J, Loo BW. Radiotherapy and immunotherapy - shining further together. JAMA Oncol 2019; 5: 1291-1292. 